\title{
A New Approach to the Application of Condition-based Maintenance Technology of Power Equipment in Smart Grid
}

\author{
Shiyu Chen ${ }^{1,}$, Yuenan Guo ${ }^{2, \text { a }}$, Wei Sun ${ }^{1, \mathrm{~b}}$, Kexin Zhang ${ }^{1, \mathrm{c}}, \mathrm{Lin} \mathrm{Li}^{1, \mathrm{~d}}$, and Long Tan ${ }^{1, \mathrm{e}}$ \\ ${ }^{1}$ State Grid Heilongjiang Electric Power Co., Ltd. Electric Power Research Institute, Harbin 150001, \\ China \\ ${ }^{2}$ State Grid Heilongjiang Electric Power Co., Ltd. Harbin 150001, China \\ auoyuenan0619@126.com, ${ }^{\mathrm{b}}$ sunwei19850525@163.com, ${ }^{\mathrm{c} 806715124 @ q q . c o m,}{ }^{\mathrm{d}}$ lilin77@yeah.net, ${ }^{\mathrm{e}}$ \\ 418337933@qq.com
}

\begin{abstract}
With the deepening of the reform of power enterprises, it is imperative for the maintenance of power equipment to be replaced by conditional maintenance. Therefore, combining the advantages of the expert system and the comprehensive analysis technology of multiple fault diagnosis algorithms, an intelligent power equipment condition maintenance system is designed. The system is mainly composed of modules such as data management, status evaluation, risk assessment, fault diagnosis, monitoring and early warning, and decision-making suggestions. Each module is independent and interconnected. Secondly, combined with the information collected by the Internet of Things to determine the maintenance plan, and then introduced the application of the Internet of Things technology in the full cycle management of power equipment, and finally compared and analysed the status maintenance and general maintenance based on the Internet of Things technology.
\end{abstract}

Keywords: Smart Grid, Power Equipment, Condition Maintenance, Internet of Things.

\section{Introduction}

With the rapid development of my country's economy, the social power load is also increasing simultaneously, the demand for electric energy is increasing, and the scale of the power grid is also expanding. At the same time, the number of various electrical equipment required in the construction of the power system is also increasing. People have higher and higher requirements for the quality of power supply, and higher requirements are put forward for the operational stability of electrical equipment. In order to ensure the stable and reliable operation of the power system, it is necessary to do a good job in the maintenance of power and electrical equipment.

At present, there are three main maintenance methods for power and electrical

*uniquecharm@live.cn 
equipment, namely passive maintenance, preventive maintenance and conditional maintenance. Among them, passive maintenance, namely post-mortem maintenance, is a typical passive maintenance mode. It is a method of maintenance after electrical equipment fails, and there is a serious lag; preventive maintenance is planned maintenance, and it is easy to cause excessive maintenance due to unreasonable planning. Insufficient overhaul and other issues, over-overhaul will result in a waste of overhaul resources, and insufficient overhaul will affect the stable operation of electrical equipment; the status-based overhaul mode is an overhaul mode based on real-time monitoring of the operating status of the equipment to take preventive measures against failures [1]. The equipment failure probability is significantly reduced and even "zero failure" can be achieved, which greatly improves the stability and reliability of electrical equipment operation. The following is an in-depth study on conditional maintenance technology.

\section{Condition maintenance}

Condition maintenance is a maintenance method based on the status of the equipment, which is different from the traditional maintenance method based on cycle (time). It is based on the current actual working conditions of the equipment. It uses advanced state monitoring and diagnosis methods, reliability evaluation methods and life prediction methods to determine the health status of the equipment, identify early signs of failure, and determine the severity and failure of the fault location. Make judgments on the development trend, and based on the analysis and diagnosis results, take the initiative to implement maintenance before the equipment performance drops to a certain level or the failure will occur [2]. It ensures the safe, economic and stable operation of the power system, and plays a huge role in the deepening reform of the current power enterprises.

Condition maintenance is a maintenance mode based on multiple disciplines, which combines detection technology, power technology, diagnosis technology and computer technology in information. With the continuous deepening of power system informatization construction, it is imperative to develop and implement power equipment condition maintenance system. These systems computerize and process the daily business processing of substation maintenance, provide equipment management, maintenance plan management, professional management, report management, Web publishing and other functions, thereby reducing the burden on staff and promoting the modern management level of the maintenance area, Realize the scientific decision-making of power equipment maintenance. The simplified working diagram of the general maintenance plan is shown in Figure 1.

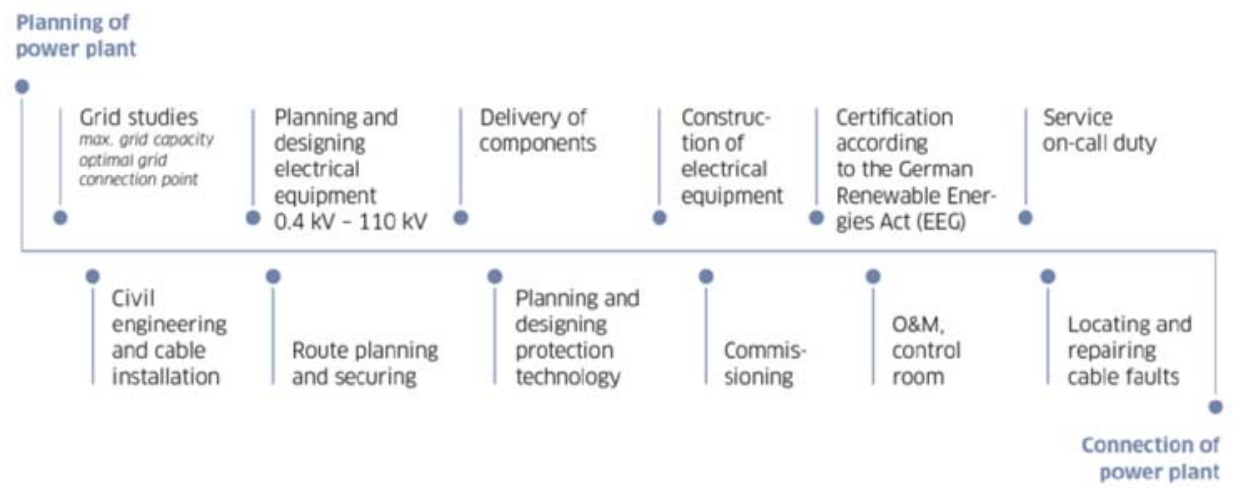

Figure 1. Simplified working diagram of condition maintenance plan 


\section{The application of loT technology in the full-cycle management of power equipment}

For the full-cycle management of power equipment, the specific applications of IoT technology are as follows: (1) Increase the initial cost of power system construction. The Internet of Things technology is an intangible commodity, so its application will inevitably cause an increase in initial costs. However, with the advent of the digital information age, this fee has been included in the cost of some basic power equipment, so its impact is small. (2) Delay the disposal of power equipment to a large extent. This article uses a large amount of space to introduce the advantages of the Internet of Things technology applied to the state maintenance of power equipment, that is, delaying the disposal of equipment scrap, improving the working status of the equipment, and reducing the possibility of power outages. (3) Reduce the consumption of manpower and financial resources. With the development of the information age, the role of labour in the power system will gradually become smaller, but the current individual labour costs are also gradually increasing, and the power industry has higher risks, resulting in more labour costs due to failures each year. The application of the Internet of Things technology greatly reduces labour costs, guarantees the safety of maintenance, and improves the quality of power supply at the same time, creating more social benefits and economic profits for power companies [3].

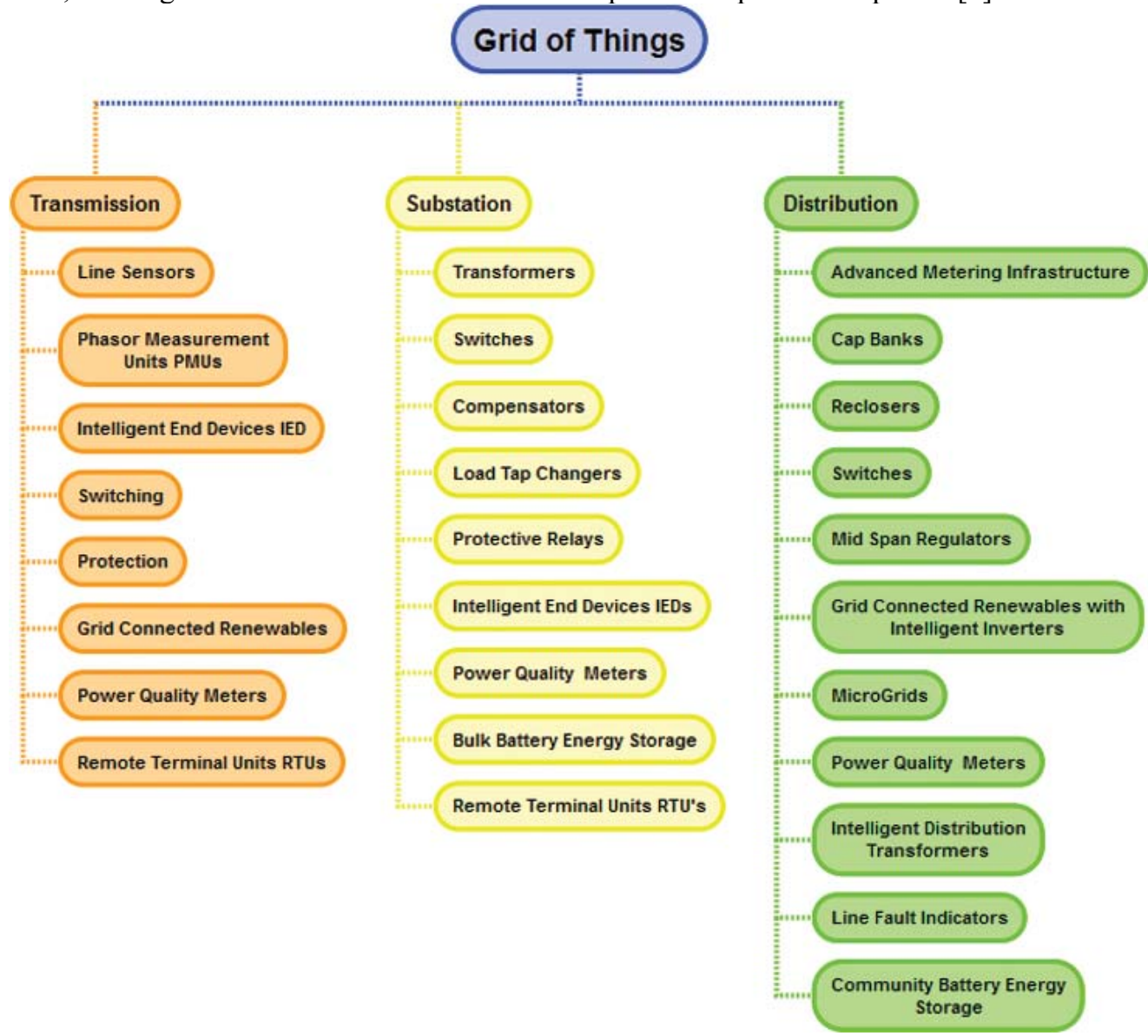

Figure 2. The structure of the power equipment condition maintenance system 


\section{Condition maintenance system design}

\subsection{System overall design}

The system is built on the remote monitoring and diagnosis centre platform system. The data management module obtains a large amount of information and data from the platform system, including equipment accounts and parameters, operation inspection records, defect records, test data, data acquisition and monitoring control (SCADA) System data, online monitoring data, etc., prepare for comprehensive intelligent analysis. Then, the system uses the expert system established by the state evaluation guidelines to conduct a comprehensive evaluation of the equipment status, evaluates the equipment with the idea of risk assessment, and activates various fault diagnosis algorithms in the library of equipment with abnormal conditions or abnormal risk assessments. The state diagnosis algorithm performs fault location and cause analysis. Finally, according to the analysis conclusions drawn from the above three steps, a monitoring and early warning is issued, decision-making suggestions on what to repair and when to repair are given, and a repair plan is made. The overall structure of the entire power equipment condition maintenance system is shown in Figure 2.

\subsection{Infrared diagnosis algorithm for power equipment}

The infrared diagnosis algorithm of power equipment includes two parts: fault judgment and fault cause diagnosis. The fault judgment link mainly judges the temperature measurement results through specific rules. I will not describe it here. The article mainly discusses the power equipment fault cause diagnosis algorithm based on case-based reasoning. Its algorithm Divided into: case matching, judgment rules and logic verification, case reuse and adaptive modification, case study, etc. The relevant case reasoning process is shown in Figure 3.

\section{The integrated fault diagnosis scheme of complex industrial process}

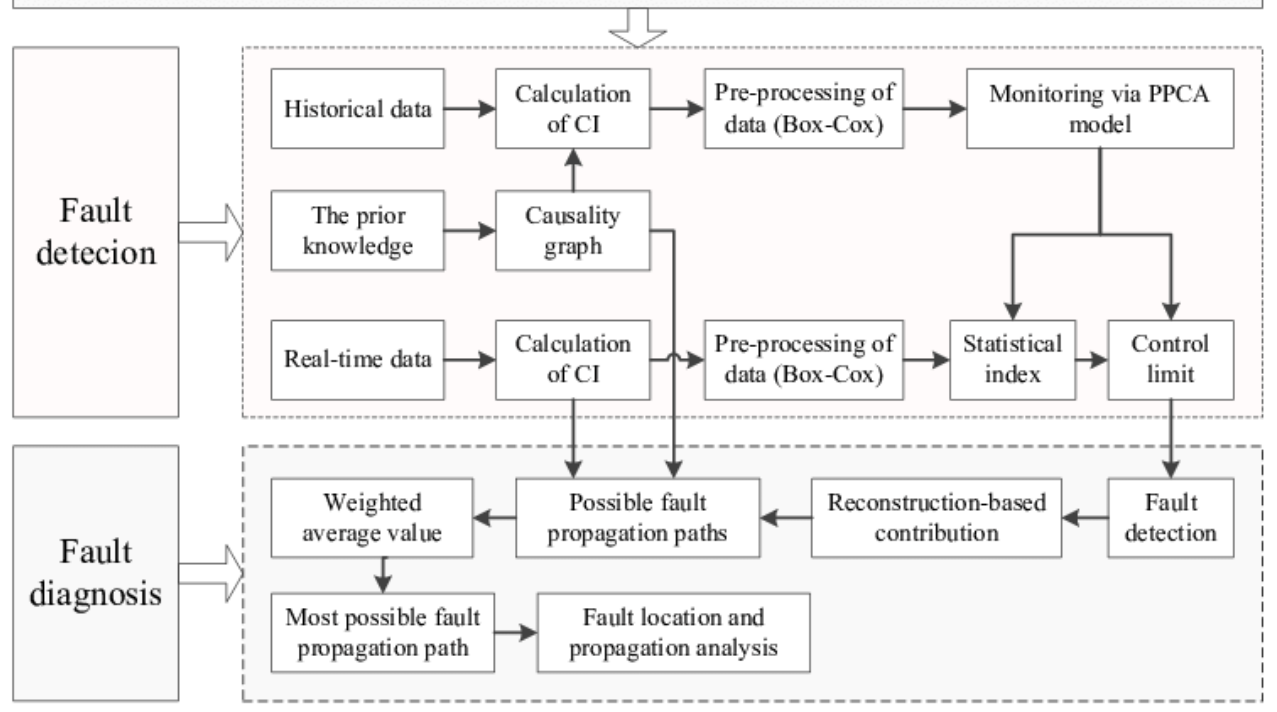

Figure 3. Case reasoning process of equipment fault diagnosis

Cases of infrared diagnosis methods are mainly obtained by collecting, sorting, and 
learning knowledge of equipment fault characteristic information, fault analysis, and handling plans, and based on the above cases, a typical equipment fault case library is established. Through the analysis of the infrared temperature measurement fault situation, it is determined that the case should include the fault characteristics, the fault description, and the description of the solution to the fault problem and the description of the solution method [4].

\subsubsection{Feature identification algorithm of feature weight.}

In order to improve the accuracy of case reasoning, a weighted screening mechanism of key features is added in the similarity comparison link. This mechanism assigns certain weights to the key characteristics shown by the instance, Expressed by vector $g_{i}$ : $g_{i}=\left(g_{i 1}, g_{i 2}, \ldots, g_{i m}\right)^{T}, I=1,2, \ldots, n$, where $\mathrm{m}$ value is the number of vectors representing the number of feature values of the case, when any feature value in $g_{i}$ is 0 , it means that the feature value is not the key feature value of this instance. The key characteristic value satisfies: $\sum_{j=1}^{m} g_{i j}=1$. The vector $x_{\beta}=\left(x_{\beta 1}, x_{\beta 2}, \ldots, x_{\beta m}\right), \beta=1,2,3, \ldots, m$ represents the infrared temperature measurement data, and $x_{\beta m}$ is the abnormal value of the m-th infrared temperature measurement junction.

\subsubsection{Similarity algorithm based on generalized distance.}

The similarity algorithm of generalized distance refers to treating similar objects as a point in an N-dimensional coordinate space. Each object represents an N-dimensional attribute value set. The smaller the space angle between the two objects, the greater the similarity. Larger, otherwise the similarity is smaller. Based on the above theory, let D denote the set of feature values of each case in the similar case set EX. The eigenvalue set of any case in $\mathrm{D}$ is represented by a data vector $\mathrm{d}$, Suppose each case $d_{\alpha} \in D(\alpha=1,2, \ldots, n)$ is represented by a vector as $d_{\alpha}=\left(d_{\alpha 1}, d_{\alpha 2}, \ldots, d_{\alpha m}\right)$, where the value of $\mathrm{m}$ is the dimension of the vector and represents the number of eigenvalues of the case, and the value of $n$ represents the number of vectors or the number of cases in the case space D. The similarity coefficient calculation formula is

$$
\operatorname{sim}\left(d_{a}, x_{\beta}\right)=c_{\alpha \beta}=\cos \left(d_{a}, x_{\beta}\right)=\frac{\left(d_{a}, x_{\beta}\right)}{\left\|d_{a}\right\| \cdot\left\|x_{\beta}\right\|}=\frac{\sum_{i=1}^{m} d_{i a}, x_{i \beta}}{\sqrt{\sum_{i=1}^{m} d_{i a}^{2} \sum_{i=1}^{m} x_{i \beta}^{2}}}
$$

\subsection{Data Management}

The main task of data management is to establish a complete data system for the system, including "static data" and "dynamic data". Static data describes the inherent characteristics of the equipment, such as ledger data and its parameters, etc.; dynamic data describes the real-time status of the equipment, such as operation inspection records, defect records, test and maintenance data, test data, bad working condition data, and SCADA system Data, online monitoring data, etc. These data are an important basis for the condition maintenance of the entire system [5]. 


\subsection{Status evaluation}

The state evaluation model is based on the expert system. It uses the expert system knowledge base compiled and compiled by famous experts in the power industry, and uses the expert system inference engine to comprehensively evaluate the operation status of the equipment. Knowledge base needs to transform state evaluation guidelines into knowledge rules, so it requires two functions: rule definition and rule analysis. The main task of the inference engine is to load the knowledge base, analyse the knowledge rules, and make inferences based on the equipment state quantity data and judgment basis cited in the knowledge base [6].

\subsection{Fault diagnosis}

Fault diagnosis enables fault location and cause analysis for equipment with abnormal status evaluation or abnormal risk evaluation. Diagnosis is not a simple process; the system needs a powerful fault diagnosis algorithm library to support. Among them, for oil chromatographic analysis data, various algorithms such as artificial neural network method, immune algorithm, modified three-ratio method, overheating discharge diagram method, David triangle method, and cubic diagram method can be activated for diagnosis and analysis, and various algorithms can complement each other. Form a comprehensive intelligent analysis. For other data, start the fault tree analysis method to analyse and judge the status of the equipment [7].

\section{Conclusion}

Power equipment condition maintenance uses the Internet of Things technology to collect accurate and objective data information, but it has no direct influence on the processing of data information and the determination of the maintenance plan. Compared with the general state maintenance, the state maintenance based on the Internet of Things technology has the advantages of efficient and intelligent maintenance work, safe and reliable maintenance process, reduced human resource consumption, and improved maintenance efficiency. Therefore, the state maintenance of power equipment based on the Internet of Things technology is further in-depth Research has important practical significance and application value.

\section{Acknowledgments}

This work was supported by Science and Technology Project of SGCC (Research on Lowtemperature Resistance Characteristics and Evaluation Technology of New Sensors for Power Transmission and Transformation).

\section{References}

1. Wang, H. Lin, D. Qiu, J. Ao, L. Du, Z. \& He, B. Research on multiobjective group decision-making in condition-based maintenance for transmission and transformation equipment based on d-s evidence theory. IEEE Transactions on Smart Grid, 6(2) (2015) 1035-1045.

2. Arab, A. Tekin, E. Khodaei, A. Khator, S. K. \& Han, Z. System hardening and condition-based maintenance for electric power infrastructure under hurricane effects. IEEE Transactions on Reliability, 65(3) (2016) 1-14. 
3. Clements, D. \& Mancarella, P. Systemic modelling and integrated assessment of asset management strategies and staff constraints on distribution network reliability. Electric power systems research, 155(2) (2018) 164-171.

4. Debnath, U. K. Ahmad, I. \& Habibi, D. Gridable vehicles and second life batteries for generation side asset management in the smart grid. International Journal of Electrical Power \& Energy Systems, 82(11) (2016) 114-123.

5. Yigit, M. Boluk, P. S. \& Gungor, V. C. A new efficient error control algorithm for wireless sensor networks in smart grid. Computer standards \& interfaces, 63(3) (2019) 27-42.

6. Nejad, H. M. Movahhedinia, N. \& Khayyambashi, M. R. Provisioning required reliability of wireless data communication in smart grid neighborhood area networks. Journal of Supercomputing, 73(2) (2017) 1-21.

7. Luis Hernández-Callejo. A comprehensive review of operation and control, maintenance and lifespan management, grid planning and design, and metering in smart grids. Energies, 12(9) (2019) 1630-1639. 